Sociologie et sociétés

\title{
Du bleu de chauffe au jean
}

Les jeux dle l'apparence des " ouvriers " à l'hôpital, entre traditions corporatistes et normes institutionnelles renouvelées

\section{From Blue-Collar Garb to Jeans}

\section{Hospital Workers' Playing with their Image, between Corporatist Traditions and Renewed Institutional Norms}

\section{Anne Monjaret}

Volume 43, numéro 1, printemps 2011

Pour une sociologie de la mode et du vêtement

For a Sociology of Fashion and Clothing

URI : https://id.erudit.org/iderudit/1003533ar

DOI : https://doi.org/10.7202/1003533ar

Aller au sommaire du numéro

Éditeur(s)

Les Presses de l’Université de Montréal

ISSN

0038-030X (imprimé)

1492-1375 (numérique)

Découvrir la revue

Citer cet article

Monjaret, A. (2011). Du bleu de chauffe au jean : Les jeux de l'apparence des " ouvriers » à l'hôpital, entre traditions corporatistes et normes institutionnelles renouvelées. Sociologie et sociétés, 43(1), 99-124. https://doi.org/10.7202/1003533ar

\section{Résumé de l'article}

Ces ouvriers revendiquent leur appartenance à l'institution, tout en n'étant pas toujours identifiés comme faisant partie de l'hôpital. Minoritaires et bien que contribuant au bon fonctionnement de l'établissement, ils sont stigmatisés dans cet univers dominé par les « blouses blanches ». Cultivant leur identité, ils disent appartenir à des corporations ouvrières aux fortes traditions. Pourtant, un effritement de ces dernières s'observe depuis des années : des métiers disparaissent, l'externalisation se développe, la figure de l'ouvrier militant n'est plus ; leur vêtement de travail change d'allure au fil du temps ; les " jeunes " s'affichent en jean, seuls les « anciens " font toujours du bleu leur emblème. Le vêtement constitue un bon analyseur des jeux et des enjeux de l'apparence, mais plus largement aussi des rapports sociaux, dans les mondes du travail.

À partir de l'analyse du « vêtement au travail » des personnels ouvriers des hôpitaux de Paris (AP-HP), Anne Monjaret cherche à saisir comment ces agents d'État, « les bleus ", se conforment ou non aux codes internes du savoir-faire et du savoir-être, comment ils échappent ou non aux normes sociales du groupe de travail ou de l'institution, comment se combinent en définitive les normes institutionnelles avec les expressions collectives - ici corporatistes - et individuelles.
Tous droits réservés @ Les Presses de l’Université de Montréal, 2011
Ce document est protégé par la loi sur le droit d'auteur. L’utilisation des services d’Érudit (y compris la reproduction) est assujettie à sa politique d'utilisation que vous pouvez consulter en ligne.

https://apropos.erudit.org/fr/usagers/politique-dutilisation/ 


\section{Du bleu de chauffe au jean}

Les jeux de l'apparence des «ouvriers» à l'hôpital, entre traditions corporatistes et normes institutionnelles renouvelées

\section{AN NE MONJARET}

CNRS, CERLIS

UMR 8070

Université Paris Descartes

45, rue des Saints-Pères

75270 Paris Cedex o6

courriel: anne.monjaret@parisdescartes.fr

\section{L}

INSTITUTION, CRÉATRICE DE RÈGLES ET DE NORMES, cherche à les maintenir et à les contrôler. Quelle est, alors, la place des corps de métier, de leur savoir-faire et de leur savoir-être dans l'affirmation d'une appartenance professionnelle au sein de l'organisation? Les salariés peuvent-ils échapper à ces normes sociales, institutionnelles et/ou corporatives? À quoi sont-ils assujettis? Comment s'y conforment-ils? Quelles sont leurs marges de manœuvre? Les mutations économiques et sociales ont-elles eu raison des modèles existants? Ce type de questionnement est loin d'être nouveau en sociologie; en revanche, ce qui apparaît plus nouveau, c'est de le mobiliser pour saisir les jeux et enjeux de l'apparence vestimentaire dans les institutions professionnelles, et ce, bien que le vêtement de travail n'ait, jusqu'à nos jours, que peu, voire incidemment ou pas, intéressé les chercheurs français en sciences humaines et sociales, l'objet, sans doute, étant trop ordinaire ou contradictoire avec la Mode (Barthes, 1967). 


\section{UN ÉTAT DE L'ART}

\section{Le vêtement de travail: une quasi-absence académique}

Le vêtement est un objet classique de la sociologie et de l'ethnologie. Pourtant, le vêtement de travail n'a pas retenu l'attention des chercheurs, en particulier en France. Ce n'est que depuis quelques années que s'observe un intérêt grandissant. Des thèses en SHS sont soutenues, par exemple sur le vestiaire des infirmières (Olivier-Amouroux, 2004) ou sur le tailleur devenu outil d'insertion aux États-Unis (Hanifi, 2006, 2008). En 2008, Ginette Francequin, psychosociologue, propose, dans un ouvrage, de le considérer comme une deuxième peau, support des sentiments d'appartenance des salariés à leur corps de métier, problématique qui rejoint la nôtre. Dans la foulée, deux colloques sont organisés, l'un, «Le vêtement de travail, une deuxième peau» en 2008 au CNAM à Paris, l'autre, «S'habiller pour travailler» en 2010 par l'Université de la Mode à Lyon.

Jusque-là, les données sont généralement éparses: analyse des habitus de classe (Bourdieu, 1979), description d'un métier ou de modes d'intégration comme l'embauche (Amadieu, 2002) apportent aux lecteurs avisés quelques indices. Pourquoi un tel désintérêt pour ce vêtement? Est-ce parce qu'il relève trop évidemment d'un ordinaire? Est-ce parce que la mode ne serait pas compatible avec les mondes du travail? Cet objet de consommation n'a pas véritablement trouvé d'écho auprès des sociologues, ni des ethnologues, alors même que Roland Barthes, en précurseur, avait posé quelques jalons dans son ouvrage Système de la Mode (1967): la rhétorique de la Mode renvoie d'abord et surtout à l'oisiveté, au festif et non au travail, celle du travail à l'hiver, à l'ennui. «Le monde de la Mode, c'est le travail en creux", écrit l'auteur (Barthes, 1967: 251). La Mode rendrait-elle invisible, à nos yeux, le vêtement fonctionnel de travail?

Il n'en est rien des chercheurs anglo-saxons ${ }^{1}$ qui se sont vite emparés de l'objet, mesurant plus rapidement que nous son intérêt, celui de faire écho à une histoire sociale du travail, des hommes et des femmes, du genre et des classes...

Dès les années 1970, des ouvrages synthétiques sur les working clothes ou l'occupational costume, traités par métier et période historique, sont publiés. Ce sont les approches historiques qui dominent, s'inscrivant plutôt dans celles classiques de l'histoire du costume (cf. Lister, 1972; Lansdell, 1977; Williams-Mitchell, 1982; Marly (de), 1986; Trunau 1994). En 1987, le Science Museum à Londres propose une exposition «Clothes for the Job" (Smart, 1987). Ce sont les uniformes scolaires, militaires, ecclésiastiques et les tenues du cow-boy, figure mythique américaine, qui ont fait couler le plus d'encre. La tradition féministe a motivé des études sur les femmes en uniforme (Ewing, 1987), les «nurses» (Brooks, Rafferty, 2007), des analyses qui prennent en compte l'adoption par les femmes de vêtements masculins, dont celui des ouvriers dans les premières décennies du xx ${ }^{\mathrm{e}}$ siècle (Williams-Mitchell, 1982; Marly (de), 1986). À partir des années 1990, les approches en communication et management apportent un point de vue plus contemporain, notamment sur l'entreprise, renouvelant les recherches sur le vêtement de

1. Nous remercions Catherine Boivin pour son aide dans la recherche de références anglo-saxonnes sur le sujet. 
travail. L'uniforme, entre conformité et transgression, semble toujours à la page (Craik, 2005). L'observation des usages vestimentaires des employées de bureau devient un objet pertinent (Rafaeli et al., 1997). Les jeux de pouvoir en exercice au travail se mesurent dans les démonstrations de l'apparence des hommes (Rucker, Anderson et Kangas, 1990) autant que des femmes (Entwistle, 1997). Les sémiologues en décortiquent les codes. La modernité des sociétés s'incarne dans le monde du secteur tertiaire et non plus du secteur secondaire.

\section{Le vêtement ouvrier masculin}

En dehors des quelques points traités par les historiens anglo-saxons du costume, le vêtement ouvrier masculin n'a pas véritablement suscité de travaux scientifiques, sociologiques et anthropologiques français, ni anglo-saxons.

En France, cependant, une première tentative de synthèse socio-économique est proposée en 1982 sur les consommations (dépense, lieu d'achat, confection personnelle) et les usages (temps et durée de leur port, usure et couture, ou neuf, et le renouvellement comme signe de distinction entre ouvriers, etc.) masculins et féminins du vêtement ouvrier (Ibarrola, 1982). Elle dégage une typologie des tenues qui distingue les vêtements de travail des vêtements de non-travail (vêtements quotidiens ordinaires et vêtements exceptionnels, de fête). Si cette étude, plutôt marxisante, ouvre les portes du vestiaire prolétaire, elle reste très sommaire et difficilement exploitable, d'autant que la critique des sources utilisées, de seconde main, est totalement absente de l'analyse. Elle présente néanmoins le mérite d'aborder le sujet.

\section{Le vêtement de travail ouvrier masculin}

Quant à la pièce maîtresse du vestiaire des ouvriers, le «bleu de travail», terme générique pour désigner les tenues de ces hommes, nos recherches en sociologie et en anthropologie sont restées vaines. Les seules données disponibles sont à glaner dans les travaux consacrés à des corps de métier, des milieux professionnels, décrivant les spécificités vestimentaires. Virginie Kollmann et Michel Valière (1992) lui consacrent un chapitre. Pour Stéphane Beaud et Michel Pialoux (1999), le vêtement de travail, emblème d'un «corps », est un indice des changements organisationnels et générationnels dans les usines Peugeot de Sochaux-Montbéliard. Mao Mollona (2002), auteur anglais, montre comment le vêtement contribue à distinguer les deux secteurs d'une entreprise de Sheffield (Grande-Bretagne) : ceux du «chaud» ne portent pas de bleu, cultivant des styles propres (chemises colorées ouvertes sur la poitrine, tee-shirts épousant leur musculature développée, survêtements ou jeans); ceux du «froid» enfilent un bleu de travail par-dessus leurs habits pour se protéger du froid et de la poussière salissante.

«Bleu» ou «jean», les deux tenues se côtoient. Sur le jean, pièce de vêtement mythique de l'ouvrier américain, la littérature ne manque pas (cf. Yonnet, 1985; Friedmann, 1987; Sullivan, 2006); elle insiste le plus souvent sur sa réappropriation par la jeunesse du monde entier à partir des années 1950-1970, comme ce fut le cas, dans une moindre mesure, de la veste pantalon Mao, dérivée du «bleu de chauffe» de l'ouvrier chinois (Yonnet, 
1985: 344). Au début des années 1970, les jeans s’affichent surchargés de motifs pour rompre avec leur habituel dépouillement puritain et remettre en cause leur production en grande série (Burgelin, 1978: 68). Le jean et l'uniforme Mao ne sont toutefois pas le bleu de chauffe des ouvriers français, ni européens comme les Italiens; et les emprunts de ce dernier par la société civile sont faits rares: dans les années 1920, un avant-gardiste italien s'en inspire pour concevoir sa «Tuta»; dans les années 1970, la jeunesse en lutte se procure des «vêtements de travail» de chez Adolphe Lafont à la Samaritaine (Chenoune, 1993).

Ce quasi vide d'études académiques sur les usages sociaux des vêtements de travail ouvriers incite à amorcer une réflexion en la matière...

Nous avons ici choisi de traiter du «vêtement au travail» ouvrier, non en partant du cas des ouvriers d'usine (Flamant et Jeudy-Ballini, 2002; Beaud et Pialoux, 1999) mais de celui des ouvriers hospitaliers de l'Assistance publique - Hôpitaux de Paris (APHP), catégorie beaucoup moins connue et étudiée. Les travaux historiques et sociologiques à leur sujet sont rares et partiels (Chevandier, 2009, 1997; Monjaret, 2008, 2006a, b, 2005a, b, 2004; Jeanjean et al., 2007; Sainsaulieu, 2006; Molinier, 2000 ; Vega, 2000; Salaün, 1999).

Ce qui fait l'intérêt sociologique de ce groupe particulier, c'est qu'il incarne les transformations subies par la catégorie professionnelle des ouvriers. Comme l'explique Éric Maurin, encore numériquement importante, cette catégorie ne se réduit pas à la seule classe ouvrière de l'industrie insérée dans des cadres productifs, elle recouvre d'autres groupes professionnels: celui des artisans de métiers (bâtiment, alimentaire, maintenance-réparation), héritiers les plus directs de l'ouvrier traditionnel de l'Ancien Régime, producteurs de biens, et celui des chauffeurs routiers, livreurs, conducteurs d'engin, magasiniers, etc., plutôt prestataires de services (Maurin, 2002: 34). Les ouvriers, hommes ou femmes, qui s'occupent de la maintenance des locaux institutionnels (universitaires, hospitaliers, muséaux...) appartiennent à ces derniers groupes, mais présentent la particularité, bien que parfois venus du privé, d'avoir intégré, par concours, le secteur public dont l'une des caractéristiques est la stabilité de l'emploi. Aujourd'hui, leur nombre s'est considérablement réduit au sein des institutions qui délèguent leurs activités au secteur privé. Ces agents qui dépendent des services techniques n'ont suscité aucun intérêt. Les ouvriers hospitaliers, et en particulier masculins, ne font pas exception. Il est temps de les prendre en considération.

C'est à l'occasion d'une recherche (1997-2004), entamée lors du transfert de trois hôpitaux pavillonnaires parisiens, Boucicaut, Broussais et Laennec (BBL), amenés à fusionner dans une nouvelle structure dite «monobloc», l'Hôpital européen GeorgesPompidou (HEGP) (Monjaret, 2008, 2005b, 2001b; Pouchelle, 2007, 2005)², que nous

2. Cette recherche, à visée ethnographique et patrimoniale, a été réalisée sous la direction de M.-C. Pouchelle, dans le cadre d'une convention «Hôpitaux en mouvement. Mémoire et ethnographie», Musée national des arts et traditions populaires (MNATP) - Centre d'ethnologie française (CEF) / Assistance publique - Hôpitaux de Paris (AP-HP). 
nous sommes penchée sur ces ouvriers de la base, hommes et non femmes - même si ces dernières ne sont pas absentes de nos analyses - et sur leurs encadrants de proximité, les maîtrises, en traitant de leur vie dans ces établissements de travail et non en dehors. Ouvriers de l' «usine» et du «bâtiment» ou jardiniers, nous nous sommes intéressée à leur fonction plus qu'à leur statut, pour établir notre choix ${ }^{3}$, une fonction qui appelle un mode vestimentaire spécifique: le bleu de travail, objet symbolique de la condition ouvrière.

Le cas de ce groupe professionnel qui oscille entre appartenance institutionnelle et appartenance corporatiste, entre visibilité et invisibilité, est pertinent afin de saisir les jeux et les enjeux de l'apparence et l'évolution des codes vestimentaires dans l'institution hospitalière. Surtout, leur situation d'exception offre un beau terrain d'observation des ajustements des normes, institutionnelles et corporatives, comme des rivalités entre corps professionnels qui cohabitent dans un même établissement, relevant d'une même institution.

Cette recherche s'inscrit dans une anthropologie des organisations et de la mémoire. Il ne s'agit pas de restituer l'histoire du vêtement de travail dans sa chronologie — d'ailleurs les souvenirs se font parfois flous à ce sujet —, mais de restituer les mémoires ouvrières et les vécus présents de ces hommes au travail, en particulier de nos informateurs privilégiés ${ }^{4}$, pour accéder à la compréhension d'un processus social, révélateur des fonctionnements institutionnels compris dans un temps long. Toutefois, avant même de s'intéresser aux tenues de travail de ces agents d'État, il nous apparaît nécessaire de cerner leur profil et leur place à l'hôpital.

\section{ÊTRE UN BLEU OU LE PROGRESSIF EFFACEMENT DE LA SCÈNE HOSPITALIÈRE}

Dans les hôpitaux de l'AP-HP, les ouvriers, agents d'État, majoritairement masculins, qui appartiennent à de multiples corps de métier, sont regroupés dans les services techniques. Ils relèvent tous d'une direction commune (Salaün, 1999) organisée selon une hiérarchisation verticale: un ingénieur ${ }^{5}$, des maîtrises, des chefs d'équipe (contremaîtres), des ouvriers d'État et des contractuels (Monjaret, 2005a). Ils sont présentés aux patients dans un livret en ces termes: «Le personnel technique et ouvrier. Vous ne le rencontrerez pas le plus souvent; il collabore pourtant activement à vos soins et à votre confort en assurant le fonctionnement et la maintenance des locaux, des appareils médicaux ou informatiques, et nombreux services de l'hôpital tels que la cuisine ou la blanchisserie» (Le livret «Votre séjour: L'AP-HP vous accueille», no 11, 2001: 5). L'institution ne semble pas les négliger.

3. Nous passerons le plus clair de notre temps dans les ateliers des services techniques, accompagnant certains agents dans leurs activités, pratiquant une observation flottante.

4. Nous avons ici surtout mis l'accent sur les paroles recueillies, sans négliger néanmoins nos observations.

5. Dans l'un des hôpitaux, l'ingénieure semble être acceptée sans problème par son équipe masculine. 


\section{Un renversement de situation: d'une visibilité positive à une visibilité négative}

Jusque dans les années 1950-1960, les ouvriers ont une place prépondérante au sein de l'hôpital (Chevandier, 2009). À l'époque, deux secteurs se distinguaient, vécus comme concurrentiels, avec ses hiérarchies (ses «aristocrates»), bien que leurs domaines d'intervention soient différents: celui dit du «bâtiment» (peintres, menuisiers, maçons, plombiers, serruriers...) sous la responsabilité d'un architecte, et celui dit de l'«usine» (chauffagistes, électriciens, mécaniciens, lampistes, techniciens du réseau téléphonique...) sous la responsabilité d'un ingénieur (Monjaret, 2005a) ${ }^{6}$. Les traces de cet organigramme se retrouvent dans les trois premiers hôpitaux enquêtés ${ }^{7}$.

Dans les années 1970, avec le départ des religieuses et l'avènement laïc, avec l'effritement des mondes ouvriers traditionnels, un changement s'amorce. Plus tard l'externalisation ${ }^{8}$, l'arrivée de nouvelles technologies vont allonger la liste des métiers traditionnels disparus. Les effectifs ouvriers connaissent une baisse significative, alors que la population totale des personnels des hôpitaux de Paris n'a cessé d'augmenter.

Cette évolution n'est pas sans conséquence sur les ouvriers, qui apparaissent désormais à l'hôpital comme un groupe minoritaire, subalterne, qui va connaître une marginalisation. La figure de l'ouvrier perd progressivement sa visibilité positive - le bleu de travail était un uniforme distinctif — pour se transformer en une visibilité négative — le bleu devient un uniforme stigmatisant —, ce qui a pour effet paradoxal de provoquer l'invisibilité de cette corporation. Les ouvriers doivent composer avec cette nouvelle donne. Déjà, dans les années 1980, la figure de l'ouvrier militant, remplacée par celle de l'infirmière militante, avait commencé à entamer leur aura dans le milieu (Ariès, 1994). Les autres corps professionnels comme les cadres semblent avoir tendance à les ignorer, les éviter ou les critiquer, les dénigrer" " "Indésirables", "rebus", "ghettos", "bras cassés", "services poubelles", etc., ces termes, utilisés par les cadres techniques, témoignent de ce que ceux-ci tendent, spontanément, à verser la présence de personnels en difficulté au compte du bilan négatif de leur service» (Molinier, 2000: 63). Pour ces cadres, la flânerie est synonyme de paresse. Les ouvriers ont conscience de leur "très mauvaise renommée», connaissent leur réputation de «déclassés». Ces représentations — publicité négative — engendrent de la souffrance au travail et sans doute plus fortement chez les anciens.

Face à une telle situation, certains expriment leurs rancœurs. L'un d'entre eux se rappelle la façon dont la direction les traitait: «il ne voulait jamais nous recevoir; nos revendications ne l'intéressaient pas. J'ai même connu une directrice adjointe ici qui

6. À l'intérieur de ce regroupement de métiers, il existe l'expression d'une hiérarchie et des «mécanismes de distinction entre les diverses spécialités (aussi en regard des matériaux manipulés)» (Jeanjean et al., 2007: 173-174).

7. «Ils ont chopé comme un virus», dit un ouvrier (mai 2004), à propos de cette tenace division corporatiste, un virus que le nouvel hôpital a tenté d'éradiquer en modifiant l'organisation des services.

8. L'arrivée d'une main-d'œuvre extérieure à l'hôpital, avec ses codes et habitudes vestimentaires, n'a-t-elle pas incité les agents à se comparer à elle, voire à la rejeter?

9. Cf. Jeanjean et al., 2007; Molinier, 2000; Vega, 2000; Peneff, 1992. 
avait dit publiquement au cours d'une réunion: «Je ne parle pas aux gens habillés en bleu». Le directeur lui a donné un congé exceptionnel pour ne plus l'avoir à l'hôpital, parce que je crois que ça aurait chauffé (...). Mais, dans la salle, il n'y avait que des gens habillés en bleu parce que c'était une réunion technique. Vous voyez, il avait gagné ce jour-là le directeur de nous envoyer quelqu'un qui ne voulait pas nous parler. C'est ça aussi des histoires de bleu, c'est une histoire» (Électricien, Boucicaut, septembre 2000). Ces souvenirs renvoient à une discrimination sociale, vécue avec violence. Des ouvriers s'élèvent d'ailleurs contre ces comportements répulsifs: "Il y a des cons partout, c'est tout. Mais, on n'a pas le droit de le dénigrer, parce qu'il est bleu, ça c'est clair» (Plombier, Broussais, décembre 1997).

Ce rejet n'est-il pas une manière détournée de ramener ce personnel à ce que l'on considère qu'il est: des «bleus", une corporation ouvrière, voire à accentuer cette caractéristique pour mieux nier leur fonction et par là leur identité hospitalière? Parmi les ouvriers rencontrés, ceux ayant intégré l'AP-HP dans les années 1960 ont été les témoins de ce processus de marginalisation, de stigmatisation qui, à terme, a eu pour effet leur effacement de la scène hospitalière. Paradoxalement leur trop grande visibilité va les rendre invisibles.

\section{Les «bleus » invisibles dans un monde dominé par les «blancs »}

Les catégories subalternes se sentent de plus en plus «transparentes»: «Je peux vous en parler en connaissance de cause, parce que, j'étais à la cuisine, j'étais en vert, et quand j'ai débuté, on m'a dit, vous savez, dans l'hôpital, il n'y a pas de sot métier. Je me suis dit, tiens! Voilà une parole sensée. Mais la semaine qui a suivi ce propos, j'étais devenu transparent. Ça, c'est le premier apprentissage, ça m’a choqué» (Magasinier, Broussais, décembre 1997).

Cette situation trouve également sa source dans les fonctions que ces agents occupent. Leur activité n'est pas à proprement parler liée aux domaines des soins, de la santé, et bien que revendiquant toujours leur appartenance à l'institution — ce sont des agents d'État —, ils ne se sentent plus identifiés ou identifiables comme faisant partie de l'hôpital.

Ils énoncent le manque de (re)connaissance, né de la perte d'un respect réciproque entre les groupes professionnels, les collègues soignants et en particulier les médecins: «Bon, les médecins, on n’en parle pas. Ils ne nous connaissent pas. Ils voient des gens passer en bleu... Dans le temps, il y avait des professeurs qui étaient complètement différents. Moi, j'en ai connu trois; il y en a un qui nous voyait, qui disait: "Bonjour Monsieur l'ouvrier". "Il s'arrêtait, il serrait la main» (Ouvrier, Broussais, juillet 1999). Ils évoquent aussi le fait que les malades et les visiteurs les ignorent: «À l'extérieur, quand quelqu'un rentre dans l'hôpital, et qu'il ne sait pas où il peut aller, où va-t-il se renseigner? À une blouse blanche. (...) et le personnel en bleu, nous, qui allons partout dans l'hôpital, qui connaissons l'hôpital comme notre poche, ils ne demandent pas. Pour les gens extérieurs, l'hôpital, c'est quelqu'un en blanc» (Plombier, Broussais, décembre 1997). Certains sont affectés par cette 
ignorance, voire cette indifférence, quand d'autres jouent avec celles-ci: «Déjà, les gens, quand tu leur dis ta profession, et que tu dis que tu travailles dans l'hôpital, les gens, ils te regardent et ils te disent: "Qu'est-ce que vous faites dans un hôpital? «(...) les gens, ils me disent: “"Il y a des menuisiers?” En plaisantant, je leur dis: “je répare les cercueils ou je répare les jambes de bois!” Pour les gens, c’est que des infirmiers, c'est du personnel en blanc» (Ouvrier, Broussais, décembre 1997). Ce besoin de justification et de retour sur un temps passé montre combien ces agents réagissent à cette forme d'invisibilité sociale quand ils n'en souffrent pas. Ces hommes sont à présent des «travailleurs de l'ombre», des travailleurs «dans l'ombre», comme ils le disent eux-mêmes ou comme le pense parfois leur hiérarchie. Autrement dit, ils sont invisibles. Est-ce parce qu'ils sont ouvriers? Parce que l'on ne souhaite pas qu'ils soient visibles? Parce qu'ils sont en inadéquation avec les représentations communes du monde hospitalier? C'est une façon de dire, de leur dire, qu'ils n'appartiennent plus ou pas à ce monde. Ce sont les «blouses blanches» qui l'incarnent. Les travaux menés par Agnès Jeanjean et son équipe confortent cette idée: «Ce sentiment de ne pas être considérés correctement, ou d'être carrément oubliés nous est apparu souvent: "Les non-soignants ont l'impression de ne pas être valorisés à l'hôpital, alors que ce sont les travailleurs de l'ombre" (Responsable du PPS). Cette citation a pris tout son sens lorsqu'au détour d'un souterrain des employés nous ont déclaré: “On n'est pas écoutés à l'hôpital, on n'est pas intéressants pour eux, même certaines personnes qui travaillent à l'hôpital croient que l'hôpital, c'est uniquement les infirmières et les médecins, mais c'est un tout. Mais nous on est dans l'ombre" (Chauffagiste).» (Jeanjean et al., 2007: 173). À leurs yeux, à l'hôpital, le monde des «blancs» apparaît plus légitime que celui des «bleus». Il est un fait: ces derniers subissent la baisse de leur effectif. Les blouses blanches, nous explique Ivan Sainsaulieu, «ont remplacé, quantitativement et qualitativement, les blouses bleues des collectifs "PO" (personnel ouvrier), dans les manifestations du corps hospitalier comme dans les représentations de la solidarité salariale à l'hôpital, solidarité salariale et solidarité humaine avec les patients n'allant pas toujours de pair» (Sainsaulieu, 2006: 82). C'est sans doute pourquoi ces derniers n'ont de cesse de se comparer aux «blouses blanches».

Ces écarts entre les deux mondes participent à la visibilité de l'un et à l'invisibilité de l'autre, et plus encore à l'énonciation d'une hiérarchisation des deux mondes. D'après les ouvriers, les «blouses blanches» figureraient en haut de l'échelle sociale tandis que les «bleus» figureraient en bas de l'échelle. Pourtant, au sein de l'hôpital, ils ne sont pas les seuls à être invisibles; par exemple les aides-soignantes que AnneMarie Arborio (2001) a étudiées le sont aussi, mais différemment parce qu'elles se placent, d'emblée, du côté des soignants.

Cette hiérarchisation prend également effet dans la répartition territoriale des deux groupes qui, si elle est plus symbolique qu'effective, reste efficace: en haut, dans la clarté, les «blouses blanches», en bas, dans les ténèbres ou les marges périphériques, 
les «bleus». Les ouvriers appartiennent à une minorité, «les bleus ${ }^{10}$ », «les blouses bleues», plutôt stigmatisée, dans un univers dominé par les «blouses blanches ${ }^{11}$ » qu'ils fréquentent rarement. Les «blouses blanches» avec qui ils entretiennent de «bonnes» relations sont les couturières, les lingères, une catégorie féminine, de la base, comme eux.

Dans le monde civil et hospitalier, cette hiérarchie est confortée par la manière dont les chercheurs, en particulier en sciences humaines et sociales, ont porté ou non leur attention sur ces corporations: la visibilité sociale et scientifique des «blouses blanches» (chirurgiens, médecins, infirmières et, même, aides-soignantes et brancardiers) contraste avec l'invisibilité sociale et scientifique des «bleus", deux sorts opposés, qui creusent l'écart de ces corps appartenant à la même institution. Pourtant des similitudes existent. À lire les résultats de Jean Peneff, les ouvriers hospitaliers pourraient présenter des points communs avec les chirurgiens qu'il étudie: «invisibilité», «sous-sol», «zone secrète et effacée de l'hôpital», "mondes clos où le personnel ordinaire n'entre pas» (Peneff, 1997: 270-271), autant d'éléments qui marquent la «frontière physique et symbolique» et qui peuvent être attribués aux deux groupes professionnels. Mais dans sa démonstration, l'auteur n'opère aucune comparaison entre les chirurgiens et les ouvriers hospitaliers. En revanche, il compare le travail minutieux des chirurgiens à celui des ouvriers artisans, des métiers manuels exercés ailleurs. En ne faisant aucune allusion aux ouvriers hospitaliers, il renforce, malgré lui, leur invisibilité, assoit leur déclassement sociologique, reflet de leur déclassement social.

«De cette invisibilité, les services techniques paient aujourd'hui le prix fort, celui de la honte et de la dévalorisation» (Molinier, 2000: 63). L'invisibilité du travail des ouvriers combinée avec l'invisibilité de la mission sociale de l'AP-HP a, sans doute progressivement, nui aux services techniques et a contribué à une incompréhension générale, elle-même nourrissant une visibilité ternie par des représentations négatives toujours prégnantes: les ouvriers restent des ouvriers dans une institution de soins et, qui plus est, ils sont «alcooliques» autant que "paresseux».

Ce groupe professionnel semble osciller entre identité ouvrière et identité hospitalière. Difficile pour les «anciens» de passer d'une figure forte de l'ouvrier militant à une image stigmatisée de l'ouvrier à la dérive; difficile d'appartenir à une institution et de s'y sentir membre à part entière tout en en étant exclus.

Le vêtement devient le symbole de ces transformations. Le bleu de travail distingue désormais par la négative, désignant toujours ceux qui appartiennent à la

10. Les groupes se distinguent à l'hôpital par des couleurs: les ouvriers en bleu, le personnel soignant en blanc, les administratifs en gris, les agents du transport en vert.

11. La blouse bleue et la blanche se distinguent aussi: «le blanc apparaissant comme une promotion sur "le bleu populaire" (Francequin, 2008: 101). «Si les femmes de ménage d'un hôpital s'étaient révoltées contre la couleur de leur blouse, bleue (trop prolétaire, trop stigmatisante), d'autres ont fini par revendiquer cette couleur symbole d'opposition au patronat» (Libération, 03/12/08: 17). Les ouvriers cultivent leur appartenance de classe alors que les femmes de ménage, sans histoire sociale collective forte, désirent se rapprocher de l'image valorisée des soignantes et s'assimiler à elles. 
famille des services techniques comme ceux qui n'y appartiennent pas ou plus. L'un d'eux raconte les effets de sa reconversion: malgré un "cursus ouvrier», "Je suis un faux bleu parce que, maintenant, je suis administratif. Et même au regard d'un bon nombre de bleus ici, je ne suis pas des leurs » (Magasinier, Broussais, décembre 1997). Mais certaines reconversions doublées de promotion peuvent être valorisées par le groupe d'origine. Lors d'une fête à laquelle nous étions conviée, un agent était fier de nous présenter un de ses anciens collègues qui ne porte plus le bleu, étant passé cadre, symbole d'une ascension sociale possible et expression d'une revanche sociale.

\section{LE VÊTEMENT DE TRAVAIL DES AGENTS: NORMES INSTITUTIONNELLES ET TRADITIONS OUVRIÈRES}

Le bleu de travail devient le symbole des transformations de l'image des services techniques. Les bleus auront du mal à se départir de cette lourde étiquette sociale, de cette situation d'invisibilité qu'ils n'ont pas toujours connue (Chevandier, 2009; 1997). En fait, ils semblent peiner depuis des années à trouver leur place dans l'hôpital.

C'est par un travail sur leur apparence qu'ils tenteront de s'en sortir, rechercheront leur légitimité, sans doute parce que le vêtement de travail est un objet qui peut être adapté tout en devant «faire avec» l'institution, car ces ouvriers hospitaliers sont des agents d'État.

\section{Un vêtement de l'institution, une tenue de protection}

L'institution a ses règles et les établissements qui en relèvent doivent les suivre; les codes de couleurs, les logos apparaissent comme une instrumentalisation du corps à leur service. Dans un document interne sur les vêtements de travail et leur usage à l'AP-HP, il est indiqué que l' «étiquette de poitrine» placée sur le vêtement de travail doit comporter le nom de l'hôpital, le nom du service, le nom de l'agent, le code hôpital et le code service, sous le porte badge. Les noms servent à l'identification du porteur et à sa reconnaissance comme agent de l'hôpital. Il s'agit de l'affichage d'une identité. Les codes à barres ou à points servent à l'identification informatique des tenues pour le nettoyage. Les ouvriers n'y échappent pas. Les cadres sont en «civil» (veste — pantalon), une façon pour eux de signifier que leur mission relève plus de l'administratif que du terrain. Les tenues sont adaptées aux activités des corps de métiers, appropriées aux tâches plus ou moins salissantes. Par exemple, les électriciens portent des chaussures de sécurité contre l'électrocution, qui, jusque dans les années 1950-1960, prenaient la forme de sabots à la semelle de bois. Selon un chef du personnel, «(...) les bleus de travail sont, effectivement, indispensables pour le personnel ouvrier qui, lui, a des tâches beaucoup plus salissantes, et cela ne serait pas normal qu'il travaille avec ses effets civils. Il porte donc le bleu de travail et les chaussures de sécurité qui sont fournis par l'AP» (Chef du personnel, Broussais, décembre 1997). 
Ces tenues fournies par l'hôpital ne sortent en principe pas de l'hôpital et restent au vestiaire ou partent à l'entretien. D’après les témoignages, dans les années 1970, les pratiques étaient plus souples, moins codifiées.

Ces vêtements sont aujourd'hui soumis à une stricte traçabilité. L'hôpital, lieu de contagion, oblige: c'est la lingerie qui procure le «matériel de travail», dresse les inventaires, repère les vêtements qui ne reviennent pas, consulte les agents concernés et s'occupe de la procédure (déclaration de perte et demande de remplacement) auprès du Service central des Blanchisseries. Tous les deux ans, les tenues des personnels sont renouvelées, à leur taille. Ce service central met à disposition des vêtements de travail avec ou sans entretien (Document interne sur l'usage des vêtements de travail, s. d.). Le rythme de change et de collecte est journalier. Dans chaque service, un agent est chargé du ramassage de linge qu'il met dans un grand sac gris portant la mention «vêtement de travail». Le linge sale ainsi récupéré est ensuite réceptionné par le service de lingerie auquel revient son lavage quand il ne l'envoie pas à la lingerie centrale (Rousset, Spadoni, 1999). À la lingerie, les couturières réparent les bleus, dépannent les ouvriers quand une fermeture éclair ou un bouton cèdent. Il arrive, cependant, que des agents rapportent chez eux leur chemise à laver, dérogeant à la règle sanitaire, car ici les risques de contagion existent et les personnels doivent se préserver.

Malgré les dangers de contamination connus en milieu hospitalier, les ouvriers resteront longtemps assez ignorants des risques encourus: si les agents peuvent constituer un vecteur pour les malades fragiles, l'inverse s'avère également. Aucune formation ne leur est cependant offerte. Dans l'un des hôpitaux étudiés, la curiosité de l'un des ouvriers, intrigué par les codes d'alerte dans les chambres des patients conduira à une revendication collective pour l'instauration de tenues de protection. La date reste floue quant à cet événement crucial qui remonte sans doute à près de 40 ans.

On peut se demander si cette ignorance n'a pas été cultivée un temps, pour entretenir la différence entre les bleus et les blancs et, surtout, confiner les ouvriers dans une image négative... Comparés aux blouses blanches, placées du côté de l'hygiène, symbole institutionnel, les ouvriers sont, eux, placés du côté du «sale», du «contagieux», situation qui ne peut qu'œuvrer à leur mise à l'écart. Leur prise de conscience de cette saleté, par exemple celle de leur tenue ou de leurs locaux, vient du regard critique développé par les autres. En revanche, ils expriment leur souci d'être "propres sur eux", forme de respectabilité de leur corps. Le port même d'un bleu contribue, cependant, à entretenir une répulsion de la part des personnels soignants, qui les met mal à l'aise quand ils ont à intervenir dans les services médicaux: "Ces casquettes-là, évidemment, on ne les prenait pas très souvent parce que, avec ça dans un service auprès du malade, ça fait un peu ridicule. Et puis, étant donné que, ce sont des bleus de chauffe, dans une chambre, vis-à-vis du malade, j'avais l'impression que c'est un peu n’importe quoi. On avait du mal quand même à supporter nos bleus de chauffe. Ça nous pesait quand même... La classe ouvrière, «biberon", etc. (...) À quoi ça nous servait la casquette? On s'en servait quand même de temps en temps. On s'en servait pour le graissage des ascenseurs. (...) Quand vous êtes sur la cabine, vous vous cognez 
un peu partout; plutôt que de se laver les cheveux tous les soirs, ce n'est pas bon non plus, eh bien on prenait la casquette. Pratiquement personne nous voyait, sur l'ascenseur ou dessous (...)» (Électricien, Boucicaut, novembre 2000). La casquette les identifie mais, selon les circonstances, les stigmatise ou les valorise. Elle a longtemps servi de signe de reconnaissance entre pairs lors des manifestations. Au moment du Front populaire, en 1936, «La casquette faisait alors partie de l'habillement normal de l'ouvrier parisien.» (Ibarrola, 1982: 21). C'est sans doute pourquoi, malgré la marque et les obligations institutionnelles, chaque bleu (dont la casquette) apparaît comme un objet personnel, et ce d'autant plus — pouvons-nous interpréter — qu'il s'agit d'un symbole.

\section{Une tradition vestimentaire ouvrière}

Toutefois, nous pouvons nous demander si, aujourd'hui, le bleu de travail n'apparaît pas davantage comme l'image d'Épinal de l'ouvrier que les anciens cultivent que comme une réalité d'usage.

Les observations font apparaître un métissage vestimentaire, chaque individu possède son style, ses façons d'associer à sa tenue de base, la veste et le pantalon, des accessoires personnels, ses manières d'être. Certains portent leur tee-shirt fétiche sans veste, quand d'autres au contraire ne laissent rien dépasser de leur bleu boutonné. Le pull vient parfois remplacer la veste, et le jean, le pantalon ou la salopette. Face aux normes institutionnelles et corporatives, les agents savent préserver leur personnalité, respectant l'équilibre accepté par le groupe de proximité, les «jeunes» se distinguent des «anciens». Le «bleu», terme générique employé pour qualifier le vêtement ouvrier, désigne également l'homme qui le porte, les personnels ouvriers, et s'inscrit dans l'histoire d'une forte tradition ouvrière.

D'ailleurs, la garde-robe de l'ouvrier hospitalier n'est pas très différente de celle de l'ouvrier de l'industrie ou de l'artisanat, ne distinguant d'ailleurs pas l'ouvrier du petit patron, artisan ou garagiste (Burgelin, 1978: 62).

Elle reste un signe distinctif qui trouve sa source dans un habitus de classe. Objet de valorisation idéologique, le bleu, et en particulier le bleu de chauffe, en soulignant une appartenance de classe, devient le symbole du «travailleur» (Kollmann, Valière, 1992: 122) dont les agents ont du mal à s'affranchir, malgré des tenues qui ont évolué.

Il faisait partie du paquetage reçu «à l'abandon» par chaque ouvrier, selon les pièces, tous les ans ou tous les deux ans. Ce paquetage se composait de bleus, de chemises, de chaussures et d'une casquette: un vestiaire type et typé (Ibarrola, 1982: 17-22). Un des informateurs nous en explique le principe: "Autrefois nous étions habillés en bleu de chauffe. Vous savez ce que c'est? [Non, mais vous allez me le dire...] Le bleu de chauffe, c'est un bleu un peu comme un bleu marine plus foncé, très foncé. Il y avait la veste, le pantalon, les chemises, il y avait plusieurs couleurs de chemises. C'est une histoire, nos bleus, bleus de travail. Tout le monde, on touche des bleus à l'abandon, c'est-à-dire qu'on nous donnait deux paires de bleus par an, avec trois chemises renouvelables tous les ans. En abandon, c'est-à-dire qu'on vous les 
donnait, vous en faisiez ce que vous en vouliez. Ils étaient à nous, et c'était fait quand même pour travailler. À cette époque-là, tout le monde s'habillait en bleu de travail, parce que les travaux, ils étaient sales. [Vous aviez deux paires de bleus, trois chemises et les chaussures]. Des chaussures de sécurité obligatoires et une paire de chaussures de ville tous les deux ans. Ça, c'était le paquetage individuel et, dans le collectif, on avait aussi quelques paires de bottes ${ }^{12}$ pour les inondations. C'était, comme ça, jusqu'en 1991» (Électricien, Boucicaut, septembre 2000). Une casquette en coton s'ajoutait à la liste, mais il ne s'en est jamais servi. Malgré ces changements, ces vêtements de travail toujours portés par les plus anciens, en âge et en ancienneté, restent une forte marque d'identité. Ils arborent encore fièrement leur bleu et leur casquette. En s'affichant avec ce symbole ouvrier, ils transcendent en quelque sorte leur appartenance hospitalière pour affirmer leur appartenance ouvrière. Ils renvoient à leur double appartenance, institutionnelle et corporatiste. Une véritable conscience de l'identité collective se forge et se revendique ainsi.

Certains nous ont demandé de les prendre en photo dans des poses significatives, tous en bleu: l'un avec sa casquette, symbole ouvrier; l'autre avec son caddie dans lequel est déposée sa boîte à outils, un objet signifiant la mobilité de son travail; un autre encore dans son atelier et, à un autre moment, le même avec le sac à linge sale dont il a la responsabilité, soulignant sa fonction. N'est-ce pas parce qu'ils ont conscience de l'effritement de leur identité traditionnelle qu'ils cherchent à l'affirmer?

Le bleu de travail devient un symbole qui se retrouve dans les décors des ateliers: ours en peluche en salopette bleue et tee-shirt à l'image de son propriétaire, calendrier d'une pin up en bleu de travail (Monjaret, 2006a et b, 2005a, 2004) ou dans les déguisements portés à l'occasion de fêtes (Monjaret, 2008, 2001a). Lors d'un pot de départ à la retraite, un agent a reçu, de l'une des couturières, un tablier bleu. Ce dernier parodie le genre ouvrier et masculin: teinté de virilité, voire de survirilité, il est, entre autres, orné d'un faux sexe proéminent et d'une pochette rouge contenant un préservatif... Les relations entre ouvriers et couturières se lisent ici: des relations professionnelles, d'amitiés et d'entraide: "C'est notre couturière. Elle fait souvent des choses comme ça, aux départs en retraite, des trucs...» (Électricien, Boucicaut, octobre 2000). Ici ce sont les personnels ouvriers, hommes et femmes qui se fréquentent.

Et comme dans l'industrie, l'identité valorisée est avant tout corporatiste. Pour Stéphane Beaud et Michel Pialoux (1999) qui se sont intéressés aux ouvriers des usines Peugeot de Sochaux-Montbéliard, les vêtements constituent des marqueurs de la classe ouvrière, d'une culture d'opposition au patronat: le bleu, vêtement du travailleur manuel, est porté avec fierté parce qu'il incarne cette culture, et pour celui qui s'en détourne, les risques d'une mise à l'écart sont grands. Il en est de même à l'hôpital: comme on nous l'a rapporté, ce peintre qui s'affiche en blouse blanche plutôt qu'en blouse bleue, non pas pour se rapprocher des soignants, mais par solidarité avec sa corporation. Mais l'excentricité marginalise: «[Pourquoi le peintre porte une blouse

12. Les bottes ont remplacé les sabots. 
blanche, lui ?] C'est une bonne question. C'est un “cas”, parmi les autres. Il est peintre. Mais c'est un peintre qui a fait — vous avez certainement entendu parler — le "compagnonnage". Il fait partie d'un groupement, les Compagnons du Tour de France. Vous connaissez ça? C'est une mentalité particulière. Lui n'a pas voulu être en bleu, parce que ce n'est pas dans les ordres de sa profession, de son groupement, et, lui, le peintre, il doit être toujours en blanc; quoi qu'il arrive, il doit être en blanc. C'est pas le blanc de la blouse. Vous avez pensé, peut-être, que c'était une blouse de... [Bah oui! je me dis: “Tiens! il est en blouse blanche comme les infirmières, les médecins!”.] Non, non, il dit: “Moi, je... je suis un artiste. Donc, je travaille en blouse”. C'est un monsieur qui vivait avec sa mère, et il faisait un peu vieux garçon» (Électricien, Boucicaut, octobre 2000). L'identité commune ouvrière n'efface pas pour autant celle plurielle des corps de métier qui revendique, au-delà leur spécialité, des valeurs propres.

\section{UN CHANGEMENT DE LOOK: SUBI OU CHOISI?}

Aujourd'hui, à l'hôpital, la discrétion du port de ces vêtements phares, qui n'ont plus qu'une valeur de témoin d'un passé révolu, en dit long sur l'impact des représentations, d'un stéréotype, dont certains vont chercher dès les années 1990 à se départir, car ils ont conscience de leur poids. L'institution elle-même propose de faire évoluer ces tenues. Que se cache-t-il derrière cette volonté de changement? Le témoignage d'un de nos informateurs privilégiés se révèle, à ce titre, précieux. Il permet de mesurer les arbitrages des ouvriers, des anciens et des jeunes, face aux nouvelles propositions.

\section{De la chemise à épaulettes au tee-shirt}

Dans les services techniques, «le look de la tenue a beaucoup changé», les chemises, bleu ciel avec des épaulettes ont longtemps été de rigueur, ce qui n'était pas au goût de tous. Certains n'aimaient pas les épaulettes, «ça leur rappelait l'armée»; beaucoup de jeunes n'y étaient pas favorables, nous a-t-on rapporté, la chemise à épaulettes faisant trop fortement référence à la culture militaire, à l'uniforme, une attitude plus générale de la jeunesse des années 1970 et 1980. Une jeunesse, soucieuse de son habillement (Ibarrola, 1982: 24) qui ne souhaite alors pas renier la culture ouvrière.

D'autres encore refuseront le port de ces chemises à cause de leur composition, en partie en Tergal. Ils craignent la transpiration. La qualité du tissu devient l'expression ouvrière du confort des corps au travail, le coton, son emblème. On comprend pourquoi cette thématique revient comme un leitmotiv dans les discours des travailleurs sur le choix de leurs tenues. Ce type d'attitude n'est pas propre aux agents hospitaliers. Nous retrouvons ces mêmes exigences et réticences dans d'autres secteurs d'activité (Ibarrola, 1982: 16). Le coton des ouvriers s'oppose au Tergal (le nylon) voulu par les patrons qui trouvent là les moyens d'une économie sur l'entretien des vêtements de travail dont ils ont la charge: avec la fibre synthétique, le lavage est facilité, le séchage plus rapide. À travers ces frictions, somme toute ordinaires, c'est le jeu 
des luttes de classes qui apparaît, ce sont les formes d'expression ouvrière qui s'affichent.

Plus tard, dans les services techniques, les chemises seront remplacées par des teeshirts. L'influence du sport sur la morphologie des vêtements de travail n'est pas nouvelle, même si elle se fait plus prégnante à certaines périodes (Yonnet, 1985: 340344). En introduisant ce type de vêtement au travail, les codes changent, les genres se brouillent: en jouant sur le sentiment de détente, l'illusion de la décontraction, l'institution ne laisse-t-elle pas à penser aux personnels que l'activité de travail est moins contraignante qu'elle n'en a l'air, que le travail croise les loisirs? Le corps, au service de l'organisation, est utilisé pour recoder les manières d'être au travail et les ouvriers ne s'y trompent pas: "Ce qui fait qu'ils préféraient les tee-shirts, parce que c'est un petit peu plus "vacances" peut-être. Ça faisait moins "officiel” que la chemise, ce qui fait que ces tenues se sont développées» (Électricien, Boucicaut, octobre 2000). Une adéquation existe entre l'offre et la demande qui explique que cette nouvelle pièce du vestiaire sera adoptée, avec succès; dans l'un des hôpitaux, cependant, la direction finit par y renoncer car, trop cher. L'économie prime: «Mais seulement, c'était plus cher. (...) plus cher, il y a un problème de budget; donc (...) sur Boucicaut (...) On nous a proposé des polos, bleus - un bleu, pas exactement comme celui-ci, mais très rapprochant» (Électricien, Boucicaut, octobre 2000). Dans ce même établissement, les changements vestimentaires se poursuivent et touchent, cette fois-ci, le vestiaire dans son ensemble.

\section{Du bleu de chauffe au bleu turquoise: changer d'apparence pour soi et les autres, une quête de reconnaissance}

En mal d'identité, en quête de reconnaissance, les services techniques supportent difficilement les stéréotypes dégradants qui leur sont habituellement associés et tentent de s'en affranchir. Ils cherchent à changer leur image grâce à diverses initiatives venant d'eux ou des responsables (ingénieur ou maîtrise). Les uns organisent des portes ouvertes ${ }^{13}$. Les autres remplacent leur traditionnel bleu de chauffe, a priori trop visible, par un bleu plus clair.

En 1989, l'AP-HP lance une opération "projet qualité» en réponse au malaise interne au sein de ses établissements. Les ouvriers de l'un des établissements décident d'y participer: «Une farouche volonté d'être mieux connus et reconnus par l'ensemble des services de l'hôpital, dont ils sont quotidiennement les prestataires, anime les personnels des services techniques usine et bâtiment (...)» (Lettre de Boucicaut, 1989: 6) et de se pencher, plus particulièrement, sur leur vêtement professionnel, objet de présentation et de représentation, pour améliorer leur «look». Une revalorisation de l'image professionnelle et, parfois, conjointement de soi, passe par une revalo-

13. Si cette initiative, démarche volontaire et mobilisatrice, a trouvé un écho positif au sein des équipes techniques, en revanche, elle n'a pas suscité la curiosité escomptée des personnels soignants restés indifférents, occasionnant une forte déception de la part des ouvriers. 
risation du paraître et par là des vêtements. Des modèles, conçus et fabriqués en interne, auraient été proposés aux ouvriers, par l'institution. Avec l'aide de la «couturière» qui a vérifié la qualité du textile, sa résistance au feu, etc., mais après de longues discussions, un vêtement de facture industrielle est retenu. Grâce à cet exemple, nous voyons encore comment se sont joués les rapports sociaux de classes: le rejet du modèle proposé par l'administration et l'adhésion collective des ouvriers à leur modèle, suivi du soutien de la direction. Nous sommes dans une institution, et les marges d'intervention autonome des personnels sont restreintes.

Au bout du compte, nous pouvons nous demander si le remplacement du traditionnel bleu par un nouveau bleu a vraiment produit les effets escomptés. L'un de nos informateurs s'en persuade: «C'est une image qui tend à s'estomper au fil du temps parce que l'image de l'ouvrier, elle, a changé. Le look de l'ouvrier, déjà, le look de l'ouvrier, et c'est quelque chose d'important...» (Électricien, Boucicaut, septembre 2000).

Nos observations montrent pourtant que, malgré ces initiatives, les «bleus » restent des «bleus», et ce, au-delà de la modification de leur tenue. Leur position de marginaux est confortée, même si certains ouvriers s'en défendent. Les ateliers et leurs occupants conservent encore une image dévalorisée et dévalorisante.

En définitive, la réussite de cette opération tient à l'engagement des ouvriers qui ont cherché à sortir du carcan traditionnel, un engagement qui s'inscrit dans une quête de légitimité. Ils ont rompu avec l'immobilisme dont ils sont taxés habituellement. C'est l'interprétation que nous en faisons et qui explique, en partie, le souci de l'un de nos informateurs à nous relater cette expérience dont il est fier, et ce, d'autant plus que les équipes d'autres hôpitaux ne s’y sont pas risquées: «Les autres sont restés avec le bleu de travail proposé par l'AP» (Électricien, Boucicaut, octobre 2000). «Vous voyez bien que ce n'était pas une couleur très reluisante... Nous avons abandonné ça en 89. Et depuis, vous voyez, c'est devenu important aujourd'hui. Tout au moins à Boucicaut. Ailleurs, certains hôpitaux sont restés longtemps avec ces bleus - et je me demande s'il n'en y a pas encore. Où l'on se rendait le mieux compte qu'ils portaient le bleu, c'est quand il y avait des manifestations devant l'avenue Victoria. Parce qu'évidemment, il y avait des représentants de chaque établissement. Donc, on voyait vraiment la différence. Quand on voyait les collègues encore en bleu de chauffe, en rapport à nos bleus, on se disait: "Mais y sont complètement arriérés!" On leur disait: "Vous n'allez pas encore faire de manifestation pour changer de bleu?" Non, ils étaient habitués comme ça...» (Électricien, Boucicaut, novembre 2000). La démonstration est claire: ces ouvriers ont été non seulement capables de changement, mais ils en ont été les acteurs, motivés par l'envie de rompre avec le stéréotype négatif dont ils sont l'objet, tout en respectant une culture corporatiste. Ils changent tout en conservant leurs valeurs d'origine.

Il reste que les mutations s'opèrent plus ou moins subrepticement. Les métiers ne sont plus ce qu'ils étaient, comme en témoigne cet électricien qui mesure les distances parcourues depuis le début de sa carrière: «Aujourd'hui, en électricité, il n’y a plus 
seulement l'électricité, on fait des choses à côté; ils font une partie d'électronique, alors que pour moi, ça n'existait pas. Vous voyez bien que de moins en moins de gens ont des bleus de travail pour travailler, beaucoup préfèrent la blouse, comme les bleus médicaux. Autrefois, un électricien faisait de tout (...)» (Électricien, Boucicaut, septembre 2000). Chaque génération apprend de l'autre génération tout en s'en distinguant. Les pratiques vestimentaires n'en constituent que le reflet.

\section{POLO ET JEAN DANS LE NOUVEL HÔPITAL: QUESTION DE GÉNÉRATION}

Le contexte de fusion des trois hôpitaux pavillonnaires dans le nouvel hôpital est particulièrement intéressant pour observer les changements de ces pratiques. Les équipes techniques ont, dans certains cas, été les dernières à partir, ce qui ne les empêche pas de se tenir au courant des projets qui les concernent, le vêtement en faisant partie. Contrairement aux changements vécus précédemment dans les anciennes structures, celui-ci apparaît plus radical aux yeux des personnels qui doivent s'adapter à la fois à de nouveaux locaux, à de nouveaux collègues, «anciens» et «jeunes», et surtout à une nouvelle organisation du travail.

En quoi le contexte a-t-il été favorable à ce changement vestimentaire? De quelle manière ce dernier se traduit-il? Les anciens vont-ils s'adapter aux nouvelles conditions? Qu'en est-il des plus jeunes?

\section{En attendant le nouveau vestiaire, des tenues transitoires entre bleu et blouse blanche}

En attendant le nouveau vestiaire, chacun porte une tenue de son choix. Avant l'arrivée dans les nouveaux locaux, l'électricien se projette: "[À l'HEGP, vous avez une autre tenue?] Non, j'ai cette tenue-là (...). Je ne mets pas la chemise avec les épaulettes parce que là-bas aussi, ils sont complètement contre. Donc, je dis: "Je ne vais pas créer de conflit entre nous, parce qu'il y a déjà suffisamment de conflits” (Électricien, Boucicaut, octobre 2000). Arrivé dans les locaux, il continue à porter son bleu et son tee-shirt au logo de Boucicaut et s'en explique: «il y a déjà des types qui sont en bleu de travail, mais en règle générale, ils viennent d'hôpitaux où les bleus étaient à l'abandon; ce qui fait qu'ils peuvent les remettre plus souvent, comme ceux de Boucicaut. C'est pour ça que, moi, je suis toujours en bleu. J'ai ramené ça de Boucicaut. Moi, il n'est pas question que je travaille en civil» (Électricien, HEGP, juillet 2001). Tous les ouvriers ne sont pas en bleu. D'ailleurs, le jour de l'inauguration de l'HEGP, le 21 décembre 2000, nous avons été surprise de ne pas remarquer distinctement les membres de l'équipe technique. Ils assistaient pourtant à la cérémonie, un certain nombre en civil ou, comme le menuisier, en blouse empruntée pour «être propre», nous a-t-il précisé plus tard.

La présence de cette blouse blanche dans les services techniques nous a intriguée. Nous avons appris qu'elle avait été proposée par la direction en attendant l'arrivée des nouvelles tenues: «Jusque maintenant, le service technique n'a pas touché de bleus de 
travail. Nous les avons reçus seulement ce matin, en partie. Ce qui fait que, par exemple, à Broussais, avant sa fermeture, les bleus n'étaient plus “à l'abandon”, ils étaient en location. Quand ils ont fermé Broussais et qu'ils sont venus ici, ils sont arrivés avec leurs habits civils: ils travaillent en habits civils. Comme c'était un peu dégueulasse quand même, ils ont été râler à la direction, la direction leur a dit: "Allez à la lingerie, on vous donnera des blouses..." Ce qui fait que mes collègues s'en allaient travailler en blouse blanche!» (Électricien, HEGP, juillet 2001). Pour cet ancien, cette blouse n'est pas adaptée à tous les types d'intervention, ce qui, à son avis, ne va pas encourager les jeunes à effectuer certaines tâches: «(...) mes collègues, ils sont très jeunes. Pour eux, le travail, c'est les ordinateurs. (...) Je dis: "il faudra bien un jour que vous lâchiez l'ordinateur et faire le travail!” Pour l'instant, ce n'était pas trop sale ce qu'on faisait, mais maintenant, ça commence... [Ils vont être obligés...] Alors, ils refusent le travail. On n'a pas ce qu'il faut... Maintenant, ils vont plus refuser (...). En blouse, c'est pas facile, vous montez des... (...) Je suis en bleu de travail, ça ne me gêne pas de faire le travail même "sale" (...)» (Électricien, HEGP, juillet 2001). Le vêtement que l'on porte serait le reflet de la façon de faire son travail.

C’est sans doute ce qui explique que cette blouse, réclamée par certains, n'ait pas été adoptée par tous. Dans l'atelier des électriciens, nous avons croisé des jeunes gens en jean et polo, les blouses blanches étant restées suspendues à une patère. Doit-on y lire une inadaptabilité du vêtement ou l'affichage d'une différence corporatiste? La blouse blanche, avec tout ce qu'elle incarne, ne serait-elle pas l'exclusive des soignants?

Ce contexte provisoire favorise peut-être les arrangements. En mars 2001, nous observions déjà dans les ateliers une absence d'homogénéité: cadres, maîtrises, magasiniers et stagiaires en civil, c'est-à-dire en veste pantalon et souvent cravate pour la hiérarchie ou en pull pantalon, ouvriers en bleu, de chauffe ou veste avec le logo AP-HP, mais la blouse n'avait pas encore fait son apparition. Chacun individu se cantonnait dans ses positions en attendant le nouveau vestiaire composé de chemises, jeans, vestes et casquettes à visière et d'un supplément de polos à manches longues en coton gris clair. Bien que le choix de ce vestiaire se soit effectué en concertation avec les personnels, il appartient en définitive au projet de l'établissement et, plus largement, de l'institution qui vise le renouvellement et la modernisation du look ouvrier. La confiance des autres corps de l'hôpital pourra-t-elle cependant être regagnée? Rien n'est moins sûr. L'institution brouille — ou du moins revisite — une nouvelle fois les codes vestimentaires au travail. En proposant des tenues sportives, urbaines et jeunes qui renvoient à l'idée de détente, ne risque-t-elle pas de renforcer le stéréotype de l'ouvrier flâneur? À moins que le jean, objet mythique, ne soit rapporté à sa fonction d'origine: un pantalon coupé dans une toile solide à l'usage des travailleurs américains (Friedmann, 1987) ou à sa fonction plus tardive d'emblème: un objet souvenir des origines de la classe ouvrière, les cols bleus. La question reste à creuser.

Il s'agit, surtout, pour la direction d'utiliser le vêtement comme instrument de rupture avec des pratiques passées, marquées par une image désuète qui ne correspond pas à celle qu'elle cherche désormais à diffuser. Les jeans doivent remplacer les bleus. 
Mais l'institution a conscience que le changement doit se faire en douceur: par exemple, les chemises appartiennent toujours au vestiaire et les vestes restent bleues: «Et les vestes! et les blousons, la commande n'est pas encore partie. Je crois qu'il y en a un au magasin, un peu comme les nôtres, d'ailleurs. On peut le voir parce que je crois bien qu'ils ont des bleus d'essai là» (Électricien, HEGP, juillet 2001). Sur le terrain, les hommes s'approprient à leur manière le vestiaire qui leur est proposé: certains l'adoptent ou font avec, quand d'autres optent pour l'inertie, ne changeant rien à leurs habitudes vestimentaires. Cette dernière attitude peut être interprétée comme une forme de résistance aux changements. Surtout, elle indique qu'il n'existe pas de cohésion sur la question de l'apparence.

\section{Les temps de la divergence: le jean, comme contre-culture de l'atelier ou le recul de l'ouvrier}

Anticipant les réactions, la direction tente de ménager les susceptibilités en impliquant les personnels dans la réflexion de cette réorganisation. Chaque concertation donne lieu à discussion et le choix des vêtements des services techniques en offre l'illustration. Malgré de telles initiatives, le choix arrêté est loin de faire l'unanimité.

Les divergences entre corps de métier resurgissent, rendant impossible l'uniformisation du vestiaire des équipes techniques. Les «bio-médicaux» rejettent la proposition des bleus de travail présentée par la direction; ils préferent conserver leur blouse, s'identifiant plutôt aux personnels de soins.

Les différences de classes apparaissent également. Pour certains ouvriers, l'utilité ne s'accorde pas à l'esthétique. Le vêtement de travail ne doit pas être trop beau, car il devient déplacé: "Elles ont pris ça, mais c'est très très joli. Et je ne me vois pas travailler avec ça. C'est “coton $100 \%$ ”, coton $100 \%$, ces tee-shirts à manches longues, un peu épais (...). Je me dis, en moi-même: "Si je mets ça pour travailler, je vais être mal à l'aise...”. Puisqu'ils ont décidé de donner ça, eh bien, je les garderai quand même pour la nuit! Ou je viendrai avec quand il ne fera pas trop froid. [Vous en avez eu deux? ] Deux, oui. Cinq pantalons! Il est prévu qu'on puisse changer un par jour» (Électricien, HEGP, juillet 2001). Les nouvelles tenues peuvent être détournées de l'usage de travail.

Enfin, les discordances vestimentaires entre générations préfigurent les bouleversements en cours dans les services techniques, au point que les ouvriers proches de la retraite cherchent à se distancier des débats internes, pour éviter sans doute d'en être affectés, et en même temps, ils ne peuvent s'empêcher de commenter les scènes du changement: "Ça a été une bagarre monstrueuse pour les histoires de blouses. Personne ne veut la même chose, certains veulent travailler en jeans, les autres veulent...» (Électricien, HEGP, juillet 2001).

La confrontation entre partisans du bleu et du jean recoupe l'opposition entre «anciens» et «jeunes». Dans cette négociation, les «anciens» semblent avoir été les grands perdants: «Je vous dis une chose: “je n’ai jamais aimé les jeans, même sans travailler" [vous avez votre réserve de jeans maintenant] Cinq! Alors, j'ai dit à mes 
collègues: "Qu'est-ce que vous en pensez? Ils sont tout neufs, ils sont pas mal! Maintenant, vous allez pouvoir travailler avec vos affaires de ville..." - parce qu'ils sont aussi en jeans — "Et vous garderez ça pour venir au travail..." (Électricien, HEGP, juillet 2001). Ces propos font ressortir l'impossible imbrication des vêtements de travail et de ville ${ }^{14}$. Le costume de ville est sorti au travail à l'occasion de manifestations d'exception, comme nous avons pu l'observer, lors des festivités officielles de fermetures ou «pots de départ» (Monjaret, 2008).

Ce témoignage montre combien le changement est difficile à accepter, s'il bouscule trop les habitudes. Dans ces conditions, conserver ses habitudes peut devenir un acte de résistance, de provocation, plutôt que de vivre le changement comme une dépossession de soi-même, de sa culture de référence.

En juillet 2002, nous notions sur notre carnet de terrain, après la visite rendue à l'un de nos informateurs privilégiés: "Aujourd'hui, il porte un tee-shirt Boucicaut et son bleu habituel. Il me raconte ce que cela signifie pour lui: c'est une façon de provoquer les cadres. Les jeans, il ne les met pas» (Carnet de terrain, HEGP, juillet 2002). $S$ 'il garde et surtout affiche les signes de son ancien établissement, Boucicaut, alors fermé, c'est pour mieux nous notifier également la difficile fusion des trois hôpitaux dans le nouveau lieu. S'il ne porte pas le jean, c'est qu'il ne tient pas à abandonner ses valeurs. La comparaison, teintée de critique, précédemment mentionnée, que cet ouvrier formulait sur le jean en général, était une manière de nous signifier l'existence d'une séparation générationnelle qui s'exprime dans des goûts vestimentaires différenciés, et au-delà aussi ${ }^{15}$ : «C'est vrai, les jeunes, ils aiment beaucoup le jean. Moi, le jean, ce n'est pas tellement de ma génération... Je m’y serais mis, peut-être, mais je trouve ça, c'est un peu rêche. (...) Moi, je n'étais pas pour le jean. Sans compter, en plus, qu'il y avait un petit liseré, au-dessus. Au bout de quatre-cinq lavages, ça s'en allait en charpie. Je trouvais ça un peu sale, quoi. Pour un hôpital, c'était pas l'idéal» (Électricien, Boucicaut, octobre 2000).

Si le jean n'est pas apprécié par les plus anciens, il l'est des jeunes qui rejettent le bleu: «Les jeunes ne veulent plus se mettre en bleu, ils ne veulent plus être reconnus dans leur fonction» et cela ne date pas d'hier: «Mais les jeunes ont du mal à..., ils avaient du mal quand même..., ils avaient du mal à accepter les bleus de chauffe (...)» (Électricien, Boucicaut, novembre 2000). Les jeunes ont adopté le jean, bien avant que l'institution ne leur propose. L'adoption du jean apparaît comme l'expression d'une contre-culture qui s'oppose à la culture ouvrière traditionnelle. Les nouvelles générations imposent, en quelque sorte, leurs normes, reniant celles des anciens. La rupture peut être consommée dès l'école. La culture de l'école devient la contre-culture de l'atelier. On est loin des observations de Paul Willis, où, au contraire, la culture de l'atelier apparaissait comme la contre-culture de l'école (Willis, 1978). C'est l'in-

14. Au moins jusque dans les années 50, les ouvriers étaient reconnaissables à leur bleu porté dehors et dans l'atelier (Burgelin, 1978: 61).

15. Les jeunes apparaissent plus opportunistes, "plus près des cadres» — ce qui peut être traduit par «trop près des cadres». 
verse qui tend désormais à se produire. Les jeunes ouvriers qu'ont rencontrés Stéphane Beaud et Michel Pialoux refusent de porter des chaussures de sécurité ou de fermer leur bleu de travail dans les ateliers du lycée, laissant apparaître un tee-shirt de couleur; ils refusent, en définitive, "de se laisser enfermer dans cet habit d'ouvrier» (Beaud, Pialoux, 1999: 172). Ils marquent non pas tant une opposition à l'institution qu'à leurs aînés et à leurs valeurs.

Pour les jeunes, l'abandon des anciennes tenues ne pose pas de problèmes, d'autant qu'ils ne se reconnaissent pas dans les valeurs corporatistes des anciens. Le recul du bleu traduit le recul des mondes traditionnels ouvriers, entamé depuis déjà plusieurs décennies: "Tout le monde voudrait être cadre, le mot ouvrier, cela passe plus. (...) Cela leur colle à la peau et c'est gênant "ouvrier”, ça gêne. "Ouvrier”, c'est désuet pour la classe dirigeante; au Front populaire, il était valorisé, il était reconnu ce mot» (Carnet de terrain, HEGP, mai 2004). La figure ouvrière a perdu toute son aura. Pour les anciens qui ont plus de 30 ans de carrière, dans ces vieilles structures publiques, il est difficile, pourtant, de rompre avec leur habitus de classe.

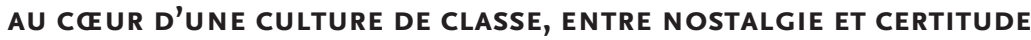

C'est avec beaucoup de nostalgie que ces anciens cultivent leur identité ouvrière et revendiquent leur appartenance à des corporations ouvrières aux fortes traditions. Les moins âgés d'entre eux ont fait avancer leur carrière et pensent à l'avenir comme les jeunes nouvellement recrutés.

Certains anciens mesurent, dès lors, qu'en voulant changer d'image, ils ont fini par se perdre. Le nouveau look ouvrier a sonné la perte d'une génération d'ouvriers. Sortir de la caverne pour retrouver une légitimité ne s'est pas fait sans conséquence. Il existe un prix de la reconnaissance. Le bénéfice d'une visibilité positive retrouvée s'est fait au détriment des signes corporatifs, repères identitaires d'hommes au travail. L'effacement de la figure de l'ouvrier est totalement consommé, et avec lui, l'effritement des corps de métier, traditionnels, du collectif ouvrier avec ses traditions et ses valeurs sur lesquelles ils s'appuyaient et se reconnaissaient. La vieille casquette du menuisier de Boucicaut dont il était si fier est reléguée dans un coin de l'atelier, accrochée au mur comme un trophée (Carnet de terrain, HEGP, juillet 2003). Même la casquette n'est plus que symbole du passé. On assiste à la crise d'une génération d'ouvriers, à celle d'une classe en perte de repères.

Cet effritement s'observe, depuis plusieurs années déjà, conséquence des transformations conjoncturelles (comme la sous-traitance, la polyvalence qui a eu pour effet la disparition progressive des métiers traditionnels) et de l'arrivée de nouvelles générations (plus mobiles, moins soucieuses des traditions ouvrières). Ce processus s'est renforcé dans le nouvel hôpital. Les changements sociaux, organisationnels et technologiques sont plus rapides que dans les anciennes structures, reconfigurant les équipes, remodelant les identités professionnelles, creusant les écarts entre les «vieux» et les «jeunes». Les premiers se projettent en arrière, quand les seconds se projettent en avant. Yvan Sainsaulieu remarque justement que, dans les hôpitaux où il a enquêté, 
les anciens valorisent le travail manuel et le collectif, tandis que les jeunes valorisent les compétences et les qualifications (Sainsaulieu, 2006: 82).

Les anciens ont la conscience des mutations et se sentent dépassés: «On travaille à l'ancienne, on part en arrière, on n'est pas adaptés à un nouveau système » (Carnet de terrain, HEGP, mai 2004). En toile de fond s'exprime le désarroi du personnel face à une nouvelle organisation du travail dont il ne maîtrise pas les rouages et qui souligne ses incompétences, l'assignant à la marginalité sur la scène du travail. Une certitude pour lui, celle de ne plus pouvoir revenir en arrière.

Le monde ouvrier hospitalier apparaît en pleine recomposition et les anciens ont du mal à trouver leur place au sein de l'institution. L'appartenance collective traditionnelle étant moins affirmée, le collectif du «PO» (personnel ouvrier) a tendance à craindre les comportements individuels (Sainsaulieu, 2006: 82) et la perte d'une dynamique consensuelle.

Du bleu de chauffe au jean, nous avons compris comment, au fil des apparences, se sont produites, au sein des hôpitaux, petites et grosses révolutions, comment les salariés ont dû jongler avec les traditions corporatistes et les normes institutionnelles sans oublier de préserver leurs individualités, comment encore le vêtement de travail et ses mises en scène ne sont que la traduction des jeux et des enjeux de pouvoir, des arrangements de classe et de genre, des mises à l'écart, entre les «blancs» et les «bleus», ou entre «anciens» et «nouveaux» («jeunes»).

Visible et invisible, ce paradoxe caractérise le groupe des agents ouvriers et, plus profondément encore, la génération des plus anciens. Il participe à un tiraillement entre son appartenance institutionnelle et son appartenance corporatiste, entre ce qu'il a été et ce qu'il est en train de devenir. Cette situation engendre une certaine souffrance au travail, du moins un désarroi.

C'est, certainement, ce contexte qui a poussé certains ouvriers à tenter de retrouver une visibilité positive grâce à un travail sur leur apparence et s'assumer comme ouvrier, en bleu. Mais ils ont fini par accentuer l'invisibilité de leur groupe, anticipant sa disparition, un groupe dont la couleur identitaire ne colle pas à celle dominante, au moins symboliquement à l'hôpital, le blanc. D'autres ont considéré leur invisibilité comme un atout: «Pour vivre heureux, vivons cachés» (Carnet de terrain, HEGP, 2003). La discrétion permet de préserver sa tranquillité au sein de l'organisation. À chacun d'envisager sa manière d'utiliser l'institution. En aucun cas, ils ne cherchent à s'identifier aux blouses blanches qui détiennent le pouvoir et la légitimité, préférant cultiver leur altérité. Quelle que soit leur tenue, ils sont et resteront des bleus.

Au-delà de ce cas de figure et en dépit des spécificités de ce groupe professionnel, n'avons-nous pas là un bel exemple de formes d'ajustements entre les injonctions institutionnelles et la voie de l'autonomie des salariés?

\section{RÉSUMÉ}

À partir de l'analyse du «vêtement au travail » des personnels ouvriers des hôpitaux de Paris (AP$\mathrm{HP})$, Anne Monjaret cherche à saisir comment ces agents d'État, «les bleus», se conforment 
ou non aux codes internes du savoir-faire et du savoir-être, comment ils échappent ou non aux normes sociales du groupe de travail ou de l'institution, comment se combinent en définitive les normes institutionnelles avec les expressions collectives - ici corporatistes - et individuelles.

Ces ouvriers revendiquent leur appartenance à l'institution, tout en n'étant pas toujours identifiés comme faisant partie de l'hôpital. Minoritaires et bien que contribuant au bon fonctionnement de l'établissement, ils sont stigmatisés dans cet univers dominé par les «blouses blanches». Cultivant leur identité, ils disent appartenir à des corporations ouvrières aux fortes traditions. Pourtant, un effritement de ces dernières s'observe depuis des années: des métiers disparaissent, l'externalisation se développe, la figure de l'ouvrier militant n'est plus; leur vêtement de travail change d'allure au fil du temps; les «jeunes » s'affichent en jean, seuls les «anciens» font toujours du bleu leur emblème. Le vêtement constitue un bon analyseur des jeux et des enjeux de l'apparence, mais plus largement aussi des rapports sociaux, dans les mondes du travail.

Mots clés: bleu de travail, hôpital, ouvrier, personnel soignant

\section{ABSTRACT}

Staring with an analysis of "work clothes" of hospital workers in Paris (AP-HP), Anne Monjaret attempts to determine whether or not these government employees, "blue collar workers," conform to internal codes of technical and interpersonal behaviour, how they manage or fail to escape the social norms of the work group or institution, and how they ultimately resolve the tension between institutional norms and collective - here corporatist and individual expressions.

These workers identify with the institution, while not always being seen as an integral part of the hospital. Although they definitely make a contribution to the good functioning of the establishment, they are a minority and are often stigmatized in this world dominated by "whitecollar" employees. Cultivating their identity, they claim to belong to longstanding workers' organizations. However, an erosion of the latter may be observed in recent years: trades are disappearing; outsourcing is becoming more popular; the figure of the worker activist has vanished; and their work clothes have changed over time - the "young people" show up in jeans and only the "old folks" continue to wear the traditional blue-collar garb. Clothing is a good indicator of the games and issues involved with image, but also, more broadly, of social relationships in the world of work.

Key words : blue collar, hospital, worker, caregivers

\section{RESUMEN}

A partir del análisis del "vestuario en el trabajo" del personal obrero de hospitales de París (Asistencia pública - Hospitales de París, AP-HP), Anne Monjaret busca comprender cómo estos agentes del Estado, "los azules", se ajustan o no a los códigos internos del saber-hacer y del saber-ser, cómo escapan o no a las normas sociales del grupo de trabajo o de la institución, cómo se combinan en definitiva las normas institucionales con las expresiones colectivas - en este caso corporativistas - e individuales. 
Estos obreros reivindican su sentido de pertenencia a la institución y, a la vez, no se identifican como parte del hospital. Minoritarios y, aun cuando contribuyen al buen funcionamiento del establecimiento, son estigmatizados en ese universo dominado por los "blusas blancas". A la vez que cultivan su identidad, dicen pertenecer a corporaciones obreras de fuertes tradiciones. Sin embargo, desde hace algunos años se observa el desmoronamiento de estos últimos: oficios que desaparecen, desarrollo de la externalización, desaparición de la figura del obrero militante; su vestimenta de trabajo cambia de apariencia con el tiempo; los "nuevos" se presentan en bluyines, tan sólo los "antiguos" hacen del azul su emblema. El vestuario constituye un buen analizador de los juegos y componentes de la apariencia pero, de forma más amplia, también de las relaciones sociales en el universo del trabajo.

Palabras claves: azul de trabajo, hospital, obrero, personal de cuidados

\section{BIBLIOGRAPHIE}

Amadieu, J.-F. (2002), Le poids des apparences: beauté, amour et gloire, Paris, Odile Jacob.

Arborio, A.-M. (2001), Un personnel invisible. Les aides-soignantes à l'hôpital, Paris, Anthropos.

ARIÈs, P. (1994), «Engagement et mise en scène: la CGT», Ethnologie française, no 4, p. 801-809.

Barthes, R. (1967), Système de la mode, Paris, Seuil.

Beaud, S. et M. Pialoux (1999), Retour sur la condition ouvrière. Enquête aux usines Peugeot de SochauxMontbéliard, Paris, Fayard.

Bourdieu, P. (1979), La distinction. Critique sociale du jugement, Paris, Éditions de Minuit.

Brooks, J. et A.-M. Rafferty (2007), «Dress and Distinction in Nursing, 1860-1939: "A corporate (as well as corporeal) armour of probity and purity”", Women's History Review, vol. 16, no 1, p. 41-57

Burgelin, O. (1978), «Du cuir, des clous et une salopette», Paris, Autrement, «Flagrants délits d'imaginaire», no 16, p. 61-71.

Chenoune, F. (1993), Des modes et des hommes. Deux siècles d'élégance masculine, Paris, Flammarion.

Chevandier C. (1997), Les métiers de l'hôpital, Paris, La Découverte.

Chevandier C. (2009), L’hôpital dans la France du xx siècle, Paris, Perrin.

Craik J. (2005), Uniforms Exposed, Oxford-New York, Berg.

Entwistle, J. (1997), «Power Dressing and the Fashioning of Carreer Woman», in M. Nava, I. Macruly,

A. Blake et R. BALY (dir.), Buy this book: Studies in Advertising and Consumption, Londres, Routledge, p. 311-324.

Ewing, E. (1987), Women in Uniform through the centuries, Londres, Sidney, Batsford.

Flamant, N. et M. Jeudy-Ballini (2002), «Le charme discret des entreprises. L'ethnologie en milieu industriel», Terrain, «Travailler à l'usine», no 39, p. 5-16.

Francequin, G. (2008), Le vêtement de travail, une deuxième peau, Ramonville-Saint-Agne, Éditions Érès.

Friedmann, D. (1987), Une histoire du blue-jean, Paris, Édition Ramsay.

Hanifi, I. (2008), «Le port du tailleur comme moyen de forger une identité de la femme au travail», Lien social et Politiques, $\mathrm{n}^{\circ}$ 59, p. 11-20.

Hanifi, I. (2006), La transmission d'un modèle de réussite à travers le vêtement. Dress for success: normalisation de la classe, de la race et du genre par l'apparence professionnelle», Thèse de doctorat, Université Paris 5 et Université de Montréal.

Ibarrola, J. (1982), S'habiller au XXe siècle (L'évolution des vêtements ouvriers), Cahiers de RHITM, no 2, Université des Sciences sociales, Grenoble, 66 p.

Jeanjean, A. et al. (2007), Une approche ethnologique des hôpitaux, Pasteur et Saint-Roch, 2005-2007, Rapport final de recherche, Centre du patrimoine/Direction des affaires culturelles de la ville de NICE, LAMIC, Université de Nice. 
Kollmann V.et M. Valière (1992), «De la blouse à la cotte», in V. Kollmann et M. Valière, Le Patrimoine industriel, Poitiers, ABCD Poitou-Charentes, Mémoires d'images, p. 121-125.

Lansdell, A. (1977), Occupational Costume and Working Clothes 1776-1976, Princes Risborough, Shire.

Lister, M. (1972), Costumes of Everyday Life: An illustrated History of Working Clothes, from 900-1910, Londres, Barrie \& Jenkins.

Marly, D. (de) (1986), Working Dress: A History of Occupational Clothing, Londres, Batsford.

Maurin, E. (2002), L'égalité des possibles. La nouvelle société française, Paris, La République des idées / Seuil. Molinier, P. (2000), "Travail et compassion dans le monde hospitalier», Cahiers du Genre, n 28, p. 49-70.

Mollona, M. (2002), "Ceux du "chaud”, ceux du "froid”. Fabriquer des outils à Sheffield», Terrain, «Travailler à l'usine», nº 39, p. 93-108.

Monjaret, A. (2008), «La fête de fermeture de trois hôpitaux parisiens, ou comment se marque, se ritualise et se fabrique l'événement», in M. BALArd et N. Gérôme, Paris et Île-de-France. Mémoires, «Les fêtes et leur histoire en Île-de-France», revue de la Fédération des sociétés historiques et archéologiques de Paris-Île-de-France, vol. 59, p. 333-356.

Monjaret, A. (2006a), «La privatisation de l'espace de travail à l'épreuve des changements professionnels et sociaux à l'hôpital», in A. BIDET et al., Sociologie du travail et activité, Toulouse, Octarès Éditions, p. 143-155.

Monjaret, A. (2006b), «Les calendriers illustrés de nus féminins dans les espaces de travail masculins», in F. Tamarozzi et D. Porparato, Oggetti e immagini. Esperienze di ricerca etnoantropologica, Université du Piémont oriental, Omega Edizioni, p. 129-156.

Monjaret, A. (2005a), «Les logiques de privatisation des ateliers techniques à l'hôpital. Se singulariser en affirmant un corporatisme», in D. BLEY, Cadre de vie et travail. Les dimensions d'une qualité de vie au quotidien, Aix-en-Provence, SEH, Édisud, p. 183-200.

Monjaret, A. (2005b), "La fermeture d'établissements publics: commande et devoir de mémoire», in B. Traimond, L'anthropologie appliquée aujourd'hui, Pessac, PUB, p. 97-108.

Monjaret, A. (2004), «Images érotiques dans les ateliers masculins hospitaliers: virilité et/ou corporatisme en crise», Mouvements, "Les hommes en crise?», n 31, p. 30-35.

Monjaret, A. (2001a), «La fête, une pratique extra-professionnelle sur les lieux du travail», Revue Cités, $\mathrm{n}^{\mathrm{o}} 8$, p. $87-100$.

Monjaret, A. (2001b), «Fermeture et transfert de trois hôpitaux parisiens. L'ethnologue, accompagnateur social», Ethnologie française, «Terrains minés», nº 1, p. 103-115.

Olivier-Amouroux, M. (2004), Chronique d'un vestiaire hospitalier, Paris, L'Harmattan.

Peneff, J. (1992), L’hôpital en urgence. Étude par observation participante, Paris, Métailié.

Peneff, J. (1997), «Le travail du chirurgien: les opérations à cœur ouvert», Sociologie du travail, no 3, p. 265-296.

Pouchelle M.-C. (2007), «Fusions d'hôpitaux, tissages d'histoire», in Y. MARec, Accueillir ou soigner? L'hôpital et ses alternatives du Moyen Âge à nos jours, Rouen, PURH, p. 95-114.

Pouchelle M.-C. (2005), «Fermeture d'hôpitaux, quelles clefs?», Ethnologie française, «Fermetures: crises et reprises ", $\mathrm{n}^{\circ} 4$, p. 593-603.

Rafaeli, A., J. Dutton, C. V. Harquail et S. Mackie-Lewis (1997), «The Use of Dress by Female Administrative Employees», The Academy of Management Journal, vol. 40, nº 1, p. 9-45.

Rousset, V. et Y. Spadoni (dir.) (1999), Des femmes, des hommes, un hôpital. Le personnel de l'AP-HP témoigne, Paris, Doin Éditeurs, Éditions de l'AP-HP.

Rucker M., E. Anderson et A. Kangas (1990), "Clothing, Power and Workplace», in K. Johnston et S. Lennon (dir.) Appearance and Power: Dress, Body, Culture, New York, Berg, p. 59-77.

SAInsaulieu I. (2006), «Les appartenances collectives à l'hôpital», Sociologie du travail, nº 48, p. 72-87.

SALAün, F. (dir.) (1999), Accueillir et soigner. L'AP-HP, 150 ans d'histoire, Paris, Doin Éditeurs, Éditions de l'AP-HP.

Smart, J.-E. (1987), Clothes for the Job, catalogue, Londres, Science Museum.

Sullivan, J. (2006), Jeans: A Cultural History of an American Icon, New York, Gotham Books. 
Trunau, I. (1994), European Occupational Dress from the Fourteenth to the Eighteenth Century, Warsaw, Institute of Archaeology and Ethnology, Polish Academy of Sciences.

VEgA, A. (2000), Une ethnologue à l'hôpital. L'ambiguïté du quotidien infirmier, Paris, Éditions des archives contemporaines.

Williams-Mitchell, C. (1982), Dressed for the Job: The Story of Occupational Costume, Poole, Dorsey, New York, Blandford Press.

Willis, P. (1978), «L'école des ouvriers», Actes de la recherche en sciences sociales, vol. 24, p. 50-61.

Yonnet, P. (1985), Jeux, modes et masses. La société française et le moderne 1945-1985, Paris, Gallimard. 